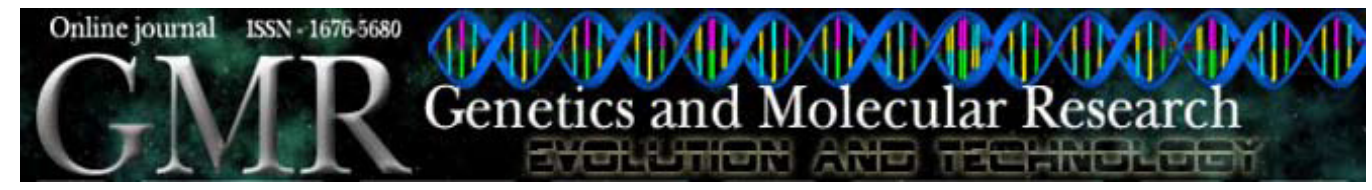

\title{
Size of the exon 1-CAG repeats of the androgen receptor gene employed as a molecular marker in the diagnosis of Turner syndrome in girls with short stature
}

\author{
C.C. Figueiredo ${ }^{1}$, C. Kochi ${ }^{1,2}$, C.A. Longui ${ }^{1,2}$, M.N. Rocha ${ }^{2}$, \\ F. Richeti ${ }^{2}$, N.M.A. Evangelista ${ }^{1}$, L.E.P. Calliari ${ }^{1}$ and O. Monte $^{1}$ \\ ${ }^{1}$ Unidade de Endocrinologia Pediátrica, Departamento de Pediatria, \\ Faculdade de Ciências Médicas, Santa Casa de São Paulo, \\ São Paulo, SP, Brasil \\ ${ }^{2}$ Laboratório de Medicina Molecular, Departamento de Fisiologia, \\ Faculdade de Ciências Médicas, Santa Casa de São Paulo, \\ São Paulo, SP, Brasil \\ Corresponding author: C.C. Figueiredo \\ E-mail: carolina-figueiredo@uol.com.br
}

Genet. Mol. Res. 7 (1): $43-49$ (2008)

Received October 8, 2007

Accepted December 20, 2007

Published January 22, 2008

\begin{abstract}
Turner syndrome (TS) is one of the most common chromosomal abnormalities among girls. Complete monosomy of X chromosome is responsible for almost 50\% of all cases of TS, and mosaicism and X anomaly are detected in the other half. It has already been demonstrated that early diagnosis of these children allows appropriate growth hormone treatment with better final height prognosis and introduction of estrogen at an ideal chronological age. Sixty-four short-stature girls were selected and the clinical data obtained were birth weight and height, weight and height at the first medical visit and target height. Other clinical data including cardiac and renal abnormalities, otitis, Hashimoto thyroiditis, cubitus valgus, short neck, widely separated nipples, and pigmented nevi were obtained from the patients' medical records. The aim of the present study was to evaluate the screening of a group of short-stature girls for TS based on the number of CAG repeats of the androgen receptor gene analyzed by
\end{abstract}


GeneScan software. Patient samples with two alleles (heterozygous) were $49 / 64$ (76.5\%) and with one allele (homozygous) were 15/64 (23.5\%). A karyotype was determined in 30 patients, 9 homozygous and 21 heterozygous. In the homozygous group, $6 / 9$ were $45, \mathrm{X}$ and $3 / 9$ were $46, \mathrm{XX}$. In the heterozygous group, $17 / 21$ were $46, \mathrm{XX}$, and $4 / 21$ were TS patients with mosaicism (45,X/46,XX; 45,X/46XiXq; 46XdelXp). The pattern obtained by GeneScan in two patients with mosaicism in the karyotype was an imbalance between the peak heights of the two alleles, suggesting that this imbalance could be present when there is a mosaicism. The frequency of TS abnormalities (18.7\%) did not differ between TS and 46,XX girls. Thus, it is important to accurately assess the incidence of TS in growthretarded girls, even in the absence of other dysmorphisms. In this study, we diagnosed 6 cases of TS 45,X (9.4\%) by molecular analysis, with a $100 \%$ sensitivity and $85 \%$ specificity. This molecular analysis was able to detect all cases of TS 45,X and the majority of mosaicisms, without the need for more $\mathrm{X}$ chromosome markers. In conclusion, determining the number of $\mathrm{CAG}$ repeats of the androgen receptor gene analyzed by GeneScan was a fast method with high sensitivity for the detection of TS 45,X, suggesting that it could be interesting as a method for screening a population of growth-retarded girls.

Key words: Turner syndrome; Androgen receptor; Molecular diagnosis; Short stature

\section{INTRODUCTION}

Turner syndrome (TS) is one of the most common chromosomal abnormalities. Its incidence varies from 1 in 1800 to 1 in 5000 live births among girls (Lippe, 1991). The prevalence at birth is about 25-55 in 100,000 girls (Stratakis and Rennert, 2005).

Complete monosomy of the $\mathrm{X}$ chromosome is responsible for almost $50 \%$ of all cases of TS (Álvarez-Nava et al., 2003). Only 20\% of TS patients are diagnosed at birth, mostly because of cardiac anomalies or hand and feet lymphedema. The remaining patients are recognized in childhood, because of short stature, or later because of pubertal delay (Massa and Vanderschueren-Lodeweyckx, 1991; Rosenfeld, 2000).

Early diagnosis of TS allows appropriate growth hormone treatment with better final height prognosis and initiation of estrogen therapy at adequate chronological age (Sävendahl and Davenport, 2000; Massa et al., 2005).

Karyotyping is the gold standard method in the diagnosis of TS, but an increasing number of molecular approaches have been described in attempt to screen TS patients among risk groups such as girls with short stature. These methods use several types and variable numbers of polymorphic X chromosome markers, allowing different detection rates of TS (Gicquel et al., 1998; Cirigliano et al., 2004).

The aim of this study was to use the number of CAG repeats of the androgen receptor (AR) gene determined by GeneScan analysis as a screening marker for TS in a group of short-stature girls. 


\section{PATIENTS AND METHODS}

Sixty-four short-stature girls were sequentially evaluated, after the informed consent form was signed by the parents or guardians. The study was approved by the Institution Ethics Committee.

Patients with other genetic, chronic or neurologic diseases with potential influence on stature were excluded.

The clinical data were obtained in the first medical visit and included weight and height, birth weight and height, and target height (TH). Anthropometric values were expressed as a standard deviation score (SDS) according to NCHS-2000 reference data (Centers for Disease Control and Prevention, 2006). TH was calculated according to the formula described by Tanner et al. (1970) and also expressed as SDS. Other descriptive data were obtained: cardiac and renal abnormalities, episodes of otitis, Hashimoto thyroiditis, cubitus valgus, short neck, widely separated nipples, and pigmented nevi.

\section{Molecular analysis}

The number of CAG repeats in the exon 1 of the AR gene is highly variable, which can be useful as a polymorphic marker. The AR gene (HUMARA gene, OMIM: 313700) is located in the Xq11-12 region. DNA samples obtained from peripheral blood lymphocytes were submitted to polymerase chain reaction (PCR) amplification, employing primers targeted to the exon 1 of the AR gene, placed externally to the CAG repeat region. The sense primer was labeled with 6-FAM (6-carboxy-fluorescein) and synthesized as follows: 5' 6-FAM - GGG TAA GGG AAG TAG GTG GAA 3' (Invitrogen Corporation, Carlsbad, CA, USA). The non-labeled antisense primer was: 5' ACT GCG GCT GTG AAG GTT 3'. The final PCR included 5 pmol of the labeled sense primer, 20 pmol of non-labeled antisense primer, $200 \mu \mathrm{mol}$ of each dNTP, $10 \mathrm{X}$ buffer, $0.1 \mathrm{U}$ Taq DNA polymerase (GeneAmp - PCR reagent kit with AmpliTaq DNA polymerase - Perkin Elmer, Branchburg, $\mathrm{NJ}$, USA), $50 \mathrm{mM} \mathrm{MgCl}{ }_{2}$, and double-distilled $\mathrm{H}_{2} \mathrm{O}$ to obtain a final volume of $50 \mu \mathrm{L}$. PCR was performed in a GeneAmp PCR System 9700 (Applied Biosystem, Foster City, CA, USA) thermocycler under the following conditions: initial denaturing step at $94^{\circ} \mathrm{C}$ for $3 \mathrm{~min}$, followed by 30 cycles of denaturation, annealing and extension $(0.5,1$ and 1 min, respectively). A final extension at $72^{\circ} \mathrm{C}$ for $7 \mathrm{~min}$ was also performed. The PCR product obtained (395-bp fragment) was then submitted to capillary gel electrophoresis in an ABI PRISM 310 automated analyzer (Applied Biosystems) under the following conditions: temperature of $60^{\circ} \mathrm{C}, 7-9 \mu \AA$ for $28 \mathrm{~min}$ for each sample. The results were analyzed employing the GeneScan software which is able to determine the relative size of the amplified fragment, where the latter variable depends on the number of CAG repeats present in the exon 1 of the AR gene. Using this method, the patients could be classified as homozygous or heterozygous depending on the size of the alleles.

\section{Statistical analysis}

Statistical analyses were performed employing the SPSS for Windows software, version 10.0.1 (SPSS Inc., Chicago, IL, USA). The Student $t$-test was used to compare variables 
between homozygous and heterozygous patients, confirmed by the karyotype 45 , X or 46,XX. The frequencies of other clinical variables, such as cardiac, renal and orthopedic diseases and Hashimoto's thyroiditis were also compared between the two groups.

\section{RESULTS}

All patients had short stature $(<-2.5$ SDS $)$ or were -2 SDS below the TH. Chronological age, height, TH, and birth weight and height from all patients are described in Table 1.

\begin{tabular}{lrr}
\multicolumn{2}{l}{ Table 1. Clinical features of short-stature girls evaluated in an endocrinology unit. } \\
\hline Clinical feature & Mean & SD \\
\hline CA at start (years) & 6.4 & 3.5 \\
Height SDS & -2.5 & 1.1 \\
TH SDS & -0.9 & 1.01 \\
Birth weight $(\mathrm{g})$ & 2544.8 & 765.7 \\
Birth height $(\mathrm{cm})$ & 44.3 & 4.9 \\
\hline
\end{tabular}

$\overline{\mathrm{CA}}=$ chronological age; $\mathrm{TH}=$ target height; $\mathrm{SDS}=$ standard deviation score.

The detection of two different alleles (heterozygous) was found in 49/64 (76.5\%) patients and only one-sized allele (homozygous) was identified in 15/64 (23.5\%) (Figure 1). The homozygous pattern usually represents the presence of a single allele, meaning that one allele is missing. Less frequently, there can be two alleles of the same size.
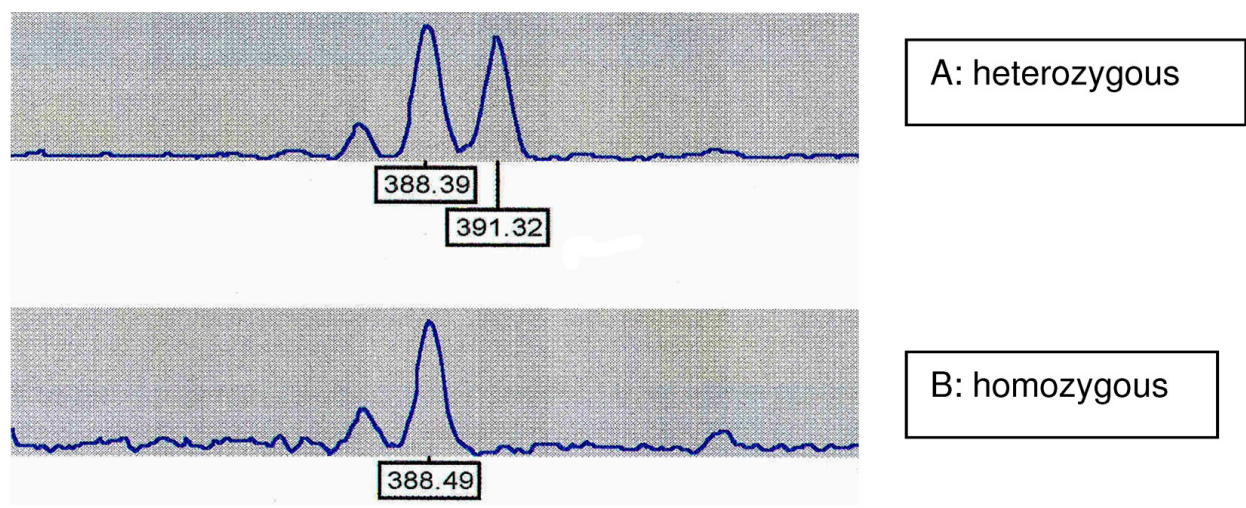

Figure 1. Electropherogram representative of one heterozygous (A) and one homozygous (B) patient.

The GeneScan data were compared to the karyotype in 30 patients. During GeneScan analyses, 9 patients were classified as homozygous and 21 as heterozygous. In the homozygous group, $6 / 9$ were 45 , X and $3 / 9$ were 46 ,XX. 45 ,X TS represented $9.4 \%$ of all short-stature girls. In the heterozygous group, 17/21 were $46, X X$, and $4 / 21$ were TS patients with mosaicism (45,X/46,XX; 45,X/46XiXq; 46XdelXp) (Table 2). Our test had a $100 \%$ sensitivity and an $85 \%$ specificity in detecting $45, X$ TS. 


\begin{tabular}{|c|c|c|c|c|c|}
\hline \multirow[t]{2}{*}{ GeneScan } & \multirow[t]{2}{*}{$\mathrm{N}$} & \multicolumn{4}{|c|}{ Karyotype } \\
\hline & & $\mathrm{N}$ & Normal & Abnormal & Karyotype \\
\hline Heterozygous & 49 & 21 & 17 & 4 & $\begin{array}{l}45, \mathrm{X} / 46, \mathrm{XX}=2 \\
45, \mathrm{X} / 46 \mathrm{XiXq}=1 \\
46 \mathrm{XdelXp}=1\end{array}$ \\
\hline Homozygous & 15 & 9 & 3 & 6 & $45, X=6$ \\
\hline Total & 64 & 30 & 20 & 10 & \\
\hline
\end{tabular}

The frequency of dysmorphic signs of TS was $18.7 \%$, but there were no significant differences between the TS patients (confirmed by karyotype) and 46,XX groups in the frequency of cardiac, renal and orthopedic abnormalities, Hashimoto's thyroiditis, or other Turner phenotypes. The frequencies of low birth weight and height SDS were also similar in the two groups.

\section{DISCUSSION}

Early detection of TS is a key factor for adequate treatment of growth disorders and other abnormalities. Growth response to growth hormone is negatively correlated with age at the start of therapy and duration of treatment (Ranke et al., 2007). Short stature is a common sign present in $98 \%$ of TS cases, even when dysmorphia is absent. In addition, early diagnosis allows the appropriate estrogen therapy and the possibility of oocyte cryopreservation.

In $18.7 \%$ of short girls, some TS phenotype signs can be present, such as cubitus valgus, short neck, widely separated nipples, and pigmented nevi. These alterations are not specific for TS, requiring stringent criteria to assess the incidence of TS among short girls, even in the absence of other dysmorphic features.

We have previously demonstrated that the GeneScan method is a sensitive and precise way to identify the parental origin of X chromosome in a 45,X TS cohort (Kochi et al., 2007), also detecting the presence of one or two $X$ chromosomes, since the AR gene is a highly polymorphic X chromosome marker.

In the general population of short-stature girls, the expected frequency of TS is supposed to be below 1\%. A study by Vimpani et al. (1981) in children between 2 and 4 years old used conventional chromosome analysis as a diagnostic test in girls with height below -2.5 SDS, and only one TS case was detected in a group of 208 short-stature girls.

Gicquel et al. (1998) diagnosed 18 (4.8\%) cases of TS in a group of 375 girls, 17 of which were detected by a molecular method (Southern blotting).

In this study, we diagnosed $6(9.4 \%)$ cases of $45, \mathrm{X}$ TS in 64 short-stature girls, detected by molecular analysis (GeneScan analysis of the polymorphic region of AR gene). This is significantly higher than the expected incidence, with a $100 \%$ sensitivity and an $85 \%$ specificity. Our institution is a tertiary hospital, and this higher incidence can be at least in part related to the selection of more severely affected girls with short stature.

It has been suggested that 45 , X monosomy is usually lethal and that most surviving $45, \mathrm{X}$ individuals are actually low-frequency mosaics undetected by classical cytogenetic tests. $45, \mathrm{X}$ monosomy is usually detected in $50 \%$ of patients with TS, and mosaicism and other X chromosome anomalies are detected in the others. According to Gicquel et al. (1998), 
mosaicism and $\mathrm{X}$ anomalies were detected in $61.1 \%$ of diagnosed TS cases. These data are consistent with the selection of patients using growth retardation as the major criterion.

Considering that GeneScan analysis is able to recognize either X chromosome monosomy or give the suspicion of mosaicism by the presence of imbalanced peaks, this method could be an interesting screening for TS patients among girls with intrauterine growth retardation or short stature. In our study, mosaicism was detected in 2 of 3 patients, because of the presence of imbalanced height of the allele peaks. Peak height imbalances are calculated in heterozygous loci. A peak height imbalance is generally considered to occur when there is a more than 30\% difference between the two peaks (Figure 2), which is larger than that observed with normal heterozygous.

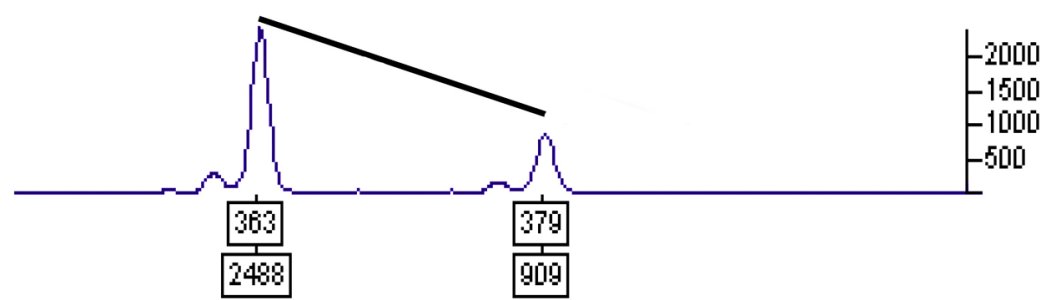

Figure 2. Electropherogram from a patient with $45, \mathrm{X} / 46, \mathrm{XX}$ karyotype, with a peak height imbalance.

This molecular approach was able to detect all cases of 45,X TS and the majority of mosaicisms, employing the variable number of CAG repeats as the only X chromosome marker. In the majority of previously reported studies, more laborious and less sensitive assays, such as Southern blotting, were employed or several polymorphic markers were needed for accurate identification (Table 3).

Table 3. Characteristics of molecular methods regarding the screening for Turner syndrome.

\begin{tabular}{|c|c|c|c|c|c|}
\hline Method & Marker & $\mathrm{N}$ & Advantages & Disadvantages & References \\
\hline Southern blotting & M27 $\beta$ & 375 & Accuracy & $\begin{array}{c}\text { Does not detect } \\
\text { mosaicism/XdelXp }\end{array}$ & Gicquel et al., 1998 \\
\hline Digestion/PCR & AR & 22 & Easy and low cost & Detects only $45, \mathrm{X}$ & Longui et al., 2002 \\
\hline QF-PCR & STR & 18,000 & Fast & Expensive & Cirigliano et al., 2004 \\
\hline Digestion/GeneScan & AR & 304 & Fast and low cost & Detects only $45, X$ & Rocha et al., 2005 \\
\hline Pyrosequencing & SNP & 25 & Detects all cases & Expensive & Meng et al., 2005 \\
\hline
\end{tabular}

$\overline{\mathrm{AR}}=$ androgen receptor; $\mathrm{STR}=$ short tandem repeats; $\mathrm{SNP}=$ single nucleotide polymorphisms; $\mathrm{QF}-\mathrm{PCR}=$ quantitative fluorescent polymerase chain reaction.

The method described in the present study was not able to detect other $\mathrm{X}$ chromosomal anomalies, especially Xp deletions, or the presence of a Y chromosome or Y-chromosome derivative material. Therefore, the inclusion of an Xp-marker and a Y-marker could expand the Y chromosome detection and recognize the increased risk of gonadal tumors.

In conclusion, the determination of the number of $\mathrm{CAG}$ repeats of the $\mathrm{AR}$ gene by GeneScan analysis is a fast and sensitive method to detect 45,X TS and potentially useful for the screening of TS patients among short girls. 


\section{ACKNOWLEDGMENTS}

We are grateful to the Support Center for Scientific Publications of Santa Casa de São Paulo, Faculty of Medical Sciences, for editorial assistance.

\section{REFERENCES}

Álvarez-Nava F, Soto M, Sánchez MA, Fernández E, et al. (2003). Molecular analysis in Turner syndrome. J. Pediatr. 142: 336-340.

Cirigliano V, Voglino G, Canadas MP, Marongiu A, et al. (2004). Rapid prenatal diagnosis of common chromosome aneuploidies by QF-PCR. Assessment on 18,000 consecutive clinical samples. Mol. Hum. Reprod. 10: 839-846.

Gicquel C, Gaston V, Cabrol S and Le Bouc Y (1998). Assessment of Turner's syndrome by molecular analysis of the X chromosome in growth-retarded girls. J. Clin. Endocrinol. Metab. 83: 1472-1476.

Kochi C, Longui CA, Lemos-Marini SH, Guerra-Junior G, et al. (2007). The influence of parental origin of X chromosome genes on the stature of patients with 45 X Turner syndrome. Genet. Mol. Res. 6: 1-7.

Lippe B (1991). Turner syndrome. Endocrinol. Metab. Clin. North Am. 20: 121-152.

Longui CA, Rocha MN, Martinho LC, Gomes GG, et al. (2002). Molecular detection of XO - Turner syndrome. Genet. Mol. Res. 1: 266-270.

Massa GG and Vanderschueren-Lodeweyckx M (1991). Age and height at diagnosis in Turner syndrome: influence of parental height. Pediatrics 88: 1148-1152.

Massa G, Verlinde F, Schepper JD, Thomas M, et al. (2005). Trends in age at diagnosis of Turner syndrome. Arch. Dis. Child. 90: 267-268.

Meng H, Hager K, Rivkees SA and Gruen JR (2005). Detection of Turner syndrome using high-throughput quantitative genotyping. J. Clin. Endocrinol. Metab. 90: 3419-3422.

Ranke MB, Lindberg A, Ferrandez Longas A, Darendeliler F, et al. (2007). Major determinants of height development in Turner syndrome (TS) patients treated with GH: analysis of 987 patients from KIGS. Pediatr. Res. 61: 105-110.

Rocha MN, Melo MR, Longui CA, de Oliveira DV, et al. (2005). A three-step molecular protocol employing DNA obtained from dried blood spots for neonatal screening for 45,X Turner syndrome. Genet. Mol. Res. 4: 749-754.

Rosenfeld RG (2000). Turner's syndrome: a growing concern. J. Pediatr. 137: 443-444.

Sävendahl L and Davenport ML (2000). Delayed diagnoses of Turner's syndrome: proposed guidelines for change. $J$. Pediatr. 137: 455-459.

Stratakis CA and Rennert OM (2005). Turner syndrome: an update. Endocrinologist 15: 27-36.

Tanner JM, Goldstein H and Whitehouse RH (1970). Standards for children's height at ages 2-9 years allowing for heights of parents. Arch. Dis. Child. 45: 755-762.

Vimpani GV, Vimpani AF, Pocock SJ and Farquhar JW (1981). Differences in physical characteristics, perinatal histories, and social backgrounds between children with growth hormone deficiency and constitutional short stature. Arch. Dis. Child. 56: 922-928. 